\title{
High expression of S100A8 and S100A9 is associated with poor disease-free survival in patients with cancer: a systematic review and meta-analysis
}

\author{
Hyun Min Koh ${ }^{1}$, Hyun Ju Lee ${ }^{2,3}$, Dong Chul Kim ${ }^{4,5,6}$ \\ ${ }^{1}$ Department of Pathology, Gyeongsang National University Changwon Hospital, Changwon, Republic of Korea; ${ }^{2}$ Department of Pathology, \\ Soonchunhyang University College of Medicine, Cheonan, Republic of Korea; ${ }^{3}$ Department of Pathology, Soonchunhyang University Cheonan \\ Hospital, Cheonan, Republic of Korea; ${ }^{4}$ Department of Pathology, Gyeongsang National University School of Medicine, Jinju, Republic of Korea; \\ ${ }^{5}$ Department of Pathology, Gyeongsang National University Hospital, Jinju, Republic of Korea; ${ }^{6}$ Gyeongsang Institute of Health Science, Jinju, \\ Republic of Korea \\ Contributions: (I) Conception and design: All authors; (II) Administrative support: HM Koh, HJ Lee; (III) Provision of study materials or patients: \\ HM Koh, DC Kim; (IV) Collection and assembly of data: HM Koh, HJ Lee; (V) Data analysis and interpretation: HM Koh, DC Kim; (VI) \\ Manuscript writing: All authors; (VII) Final approval of manuscript: All authors. \\ Correspondence to: Hyun Ju Lee, MD, PhD. Department of Pathology, Soonchunhyang University Cheonan Hospital, Soonchunhyang \\ University College of Medicine, 31 Soonchunhyang 6-gil, Cheonan 31151, Republic of Korea. Email: drlhjmd@daum.net; Dong Chul Kim, \\ MD, PhD. Department of Pathology, Gyeongsang National University School of Medicine, 79 Gangnam-ro, Jinju 52727, Republic of Korea. \\ Email: kdcjes@gmail.com.
}

Background: The expression of S100A8 and S100A9 is found to be related with the survival of cancer patients, but the results and information regarding their prognostic significance are inconsistent in literature. This study, therefore, aimed to perform a comprehensive meta-analysis and determine the prognostic significance of S100A8 and S100A9 expression in patients with cancer.

Methods: Data were collected by performing a literature search on the PubMed, Cochrane library, and Scopus databases. Pooled hazard ratio (HR) with confidence interval (CI) was calculated to assess the correlation between S100A8 and S100A9 expression and survival in patients with cancer.

Results: In total, 5 studies that enrolled 735 cancer patients were included in the meta-analysis. The pooled HR concerning S100A8 and S100A9 expression was 1.98 (95\% CI: 1.20-3.29, P=0.008) for diseasefree survival (DFS), indicating an association between high expression of S100A8 and S100A9 and poor DFS in cancer patients. However, the expression of S100A8 and S100A9 was not significantly correlated with disease-specific survival (HR 1.71, 95\% CI: 0.86-3.40, P=0.128).

Discussion: The current meta-analysis revealed that S100A8 and S100A9 expression may be a potential prognosticator of DFS in cancer patients. The present systematic review is the first to investigate the survival outcomes of cancer patients with S100A8 and S100A9 expression.

Keywords: Neoplasms; meta-analysis; S100A8; S100A9; prognosis

Submitted Mar 23, 2021. Accepted for publication Jun 16, 2021.

doi: $10.21037 /$ tcr-21-519

View this article at: https://dx.doi.org/10.21037/tcr-21-519

\section{Introduction}

Cancer is a major public health issue (1). In 2018, more than 18 million new cases and 9.5 million cancer-associated deaths were reported (2). Despite the vigorous efforts and advances in cancer management and therapy, the clinical outcome of cancer patients remains poor $(1,2)$. Thus, it is essential to develop valuable biomarkers for cancer that could improve treatment efficacy and evaluate the prognosis 
of cancer patients $(2,3)$.

S100 belong to the family of EF-hand calcium-binding proteins (4). Thus far, more than 20 members have been identified (4). They are involved in rheumatoid arthritis, inflammatory bowel disease, and other inflammatory diseases as well as in physiological functions, such as chemo-attraction for leukocytes and macrophages, calcium homeostasis, protein phosphorylation, and regulation of enzyme activity (5-7). Moreover, recent studies have suggested that alterations in the function and expression of S100 proteins may represent an essential step during cancer development and progression (4).

S100A8 and S100A9 are members of the S100 family and known to function as a heterodimer, calprotectin $(5,8)$. The S100A8 and S100A9 proteins are overexpressed in various cancers, such as melanoma, lung, gastric, colon, prostate, ovarian, head and neck, and breast cancers (8). Moreover, S100A8 and S100A9 enhance cancer cell proliferation, invasion, and metastasis (9-11).

However, the results regarding the prognostic significance of S100A8 and S100A9 expression remain controversial. Therefore, we performed a comprehensive meta-analysis to determine the prognostic significance of S100A8 and S100A9 expression in patients with cancer. We present the following article in accordance with the PRISMA reporting checklist (available at https://dx.doi. org/10.21037/tcr-21-519).

\section{Methods}

\section{Search strategy}

Studies published in literature up to January 2020 were identified through a search on the Scopus, PubMed, and Cochrane Library databases using the following search terms: (S100A8 or S100A9 or calprotectin) and (cancer or tumor or carcinoma or neoplasm or malignancy) and (prognostic or predict or prognosis or survival or outcome). All significant publications in the references of the reviewed articles were also manually evaluated.

\section{Inclusion and exclusion criteria}

The studies were required to meet the following criteria for inclusion: (I) the expression of detection of S100A8 and S100A9 in the tumor cells of human cancer tissues was assessed using immunohistochemistry (IHC); (II) relationship of S100A8 and S100A9 expression with the clinical outcome was assessed; (III) hazard ratio (HR) and $95 \%$ confidence interval (CI) evaluating multivariate regression model for clinical outcome were provided. Articles were excluded from further consideration if they met the following criteria: (I) duplicate studies; (II) reviews, case reports, letters, conference abstracts, and non-English articles; (III) preclinical studies, such as laboratory or in vitro studies; (IV) studies of patients and children with hematological malignancy; and (V) studies with unavailable survival data for further calculations (including survival curves yet without HR reported).

\section{Data extraction}

Data for the following variables were extracted from the selected articles: first author, publication year, country, cancer type, demographic characteristics (number of patients, sex, and mean or median age), endpoints, followup period, study period, S100A8 and S100A9 expression associated with poor prognosis, cut-off value for S100A8 and S100A9 expression, and reported values for HR and $95 \%$ CI for survival. Eligibility of the studies for inclusion was assessed by two authors individually, with any discordance being revised and re-evaluated.

\section{Quality assessment}

The Newcastle-Ottawa Scale (NOS) was used for estimating the quality of the eligible studies. The scale covered study group selection, comparability, and outcome ascertainment, and the scores ranged from 0 to 9 . Articles that had a score greater than 6 were considered high quality. Two authors independently performed quality evaluation for each study.

\section{Statistical analysis}

The current meta-analysis was performed using StataSE12 (Stata, College Station, TX, USA). To investigate the prognostic significance of S100A8 and S100A9 expression, pooled $\mathrm{HR}$ and $95 \%$ CI were calculated and applied. Cochran's Q and $\mathrm{I}^{2}$ statistics were used to assess heterogeneity among the selected studies. $\mathrm{I}^{2}>50 \%$ or $\mathrm{P}<0.1$ was regarded as statistically significant in a random-effects model. Insignificant heterogeneity was applied using the fixed-effects model. A subgroup analysis was conducted to determine the source of heterogeneity. Funnel plot and Egger's regression test were also performed to reveal publication bias. Sensitivity analysis was conducted to check 


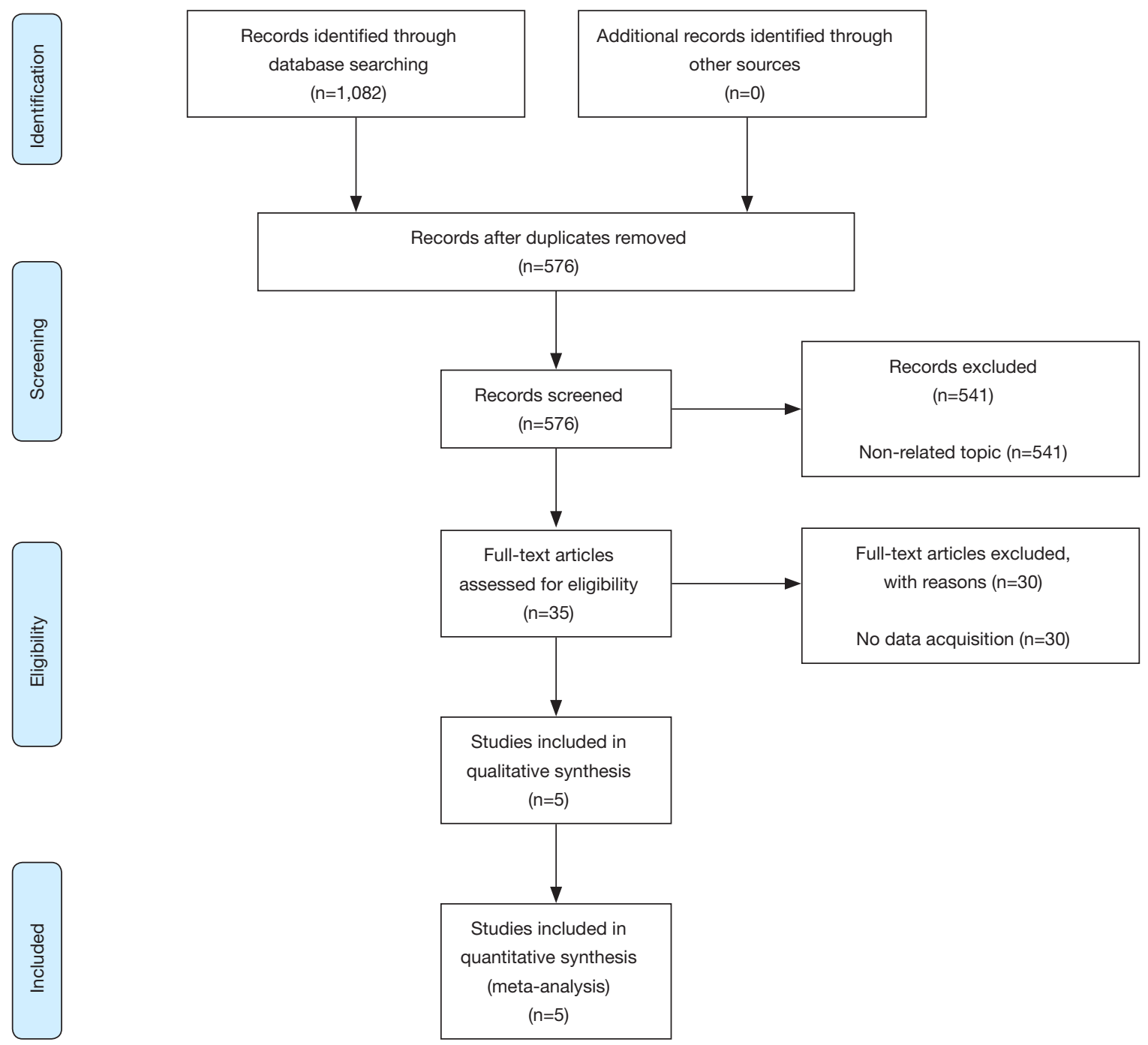

Figure 1 Flow diagram of the study selection process.

the stability of the initial results. $\mathrm{P}<0.05$ was considered to indicate statistical significance.

\section{Results}

\section{Characteristics of the included studies}

As shown in Figure 1, five studies were selected from 576 articles initially searched for current meta-analysis. The main features of the included studies are presented in Table 1. There were 735 cancer patients; therefore, 37 was the minimum sample size and 158 was the largest sample size. The enrolled studies covered five types of cancers: renal cell carcinoma $(n=2)$, lung squamous cell carcinoma $(\mathrm{n}=1)$, lung adenocarcinoma $(\mathrm{n}=1)$, breast cancer $(\mathrm{n}=1)$, and non-muscle-invasive bladder cancer $(\mathrm{n}=1)$. All the enrolled studies performed IHC to detect S100A8 and S100A9 expression in the tumor cells of human cancer tissue. The HR and $95 \%$ CI were corrected from the original articles calculated using multivariate analysis. The NOS score of all included studies was 8 .

\section{Association between S100A8 and S100A9 expression and disease-specific survival}

Five studies that included cancer patients reported the relationship of S100A8 and S100A9 expression with cancerspecific survival (CSS), disease-specific survival (DSS), and 







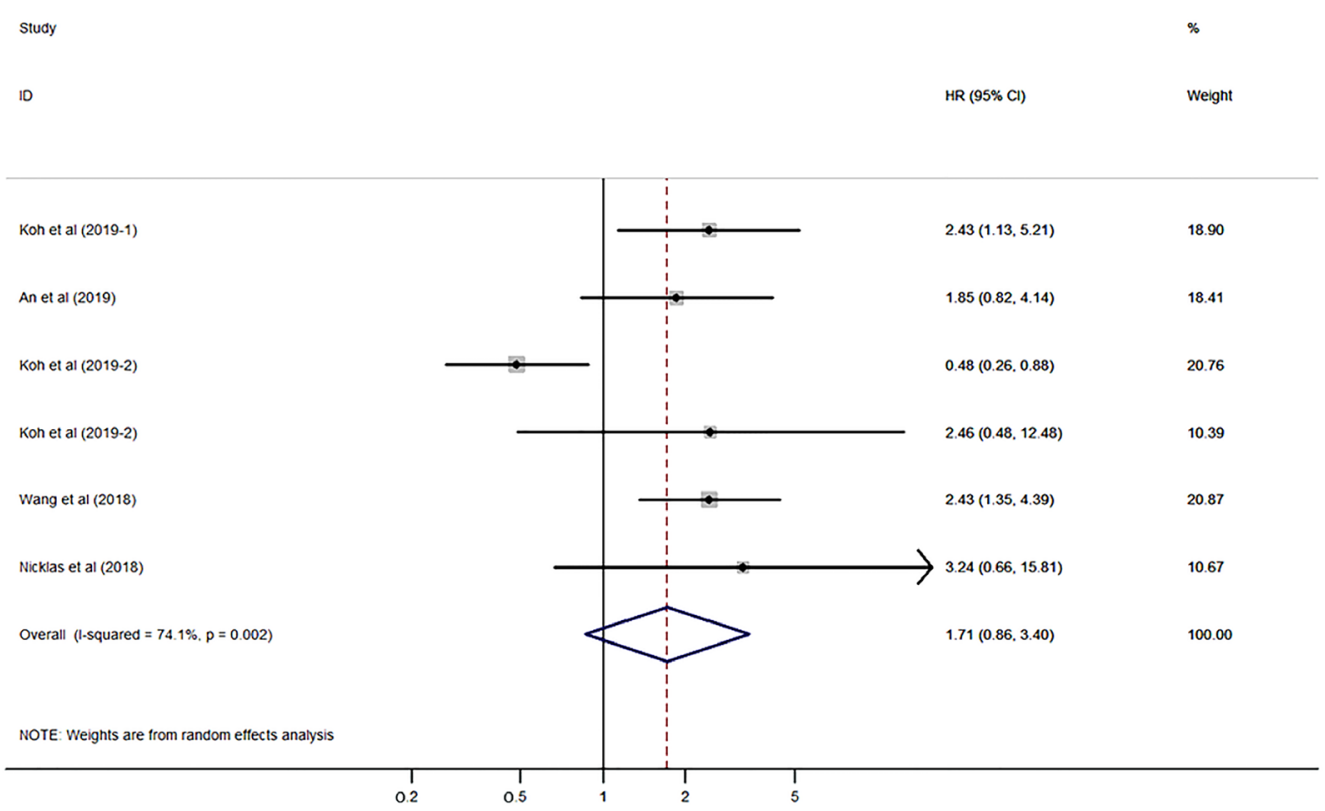

Figure 2 Forest plot presenting the association of S100A8 and S100A9 expression with disease-specific survival in cancer patients.

overall survival (OS). Koh et al. (12) reported the HRs in squamous cell carcinoma and adenocarcinoma of the lung. In this meta-analysis, CSS and OS were regarded as DSS, and the HRs reported by Koh et al. (12) in both types of lung cancer were included.

The pooled HR was evaluated using the randomeffects model due to severe heterogeneity $\left(\mathrm{I}^{2}=74.1 \%\right.$, $\mathrm{P}=0.002)$. The expression of S100A8 and S100A9 was not significantly correlated with DSS (HR $1.71,95 \%$ CI: $0.86-3.40, \mathrm{P}=0.128$; Figure 2) ( $\mathrm{P}$ value is not shown in the figure). Subgroup analysis, which was performed to reveal the cause of heterogeneity, revealed that the main sources of heterogeneity could be the protein type ( $\mathrm{S} 100 \mathrm{~A} 9, \mathrm{I}^{2}=83.3 \%$, $\mathrm{P}=0.003$ ), cancer type (non-urinary system cancer, $\mathrm{I}^{2}=86.7 \%, \mathrm{P}=0.001$ ), and sample size (sample size fewer than $100, \mathrm{I}^{2}=70.5 \%, \mathrm{P}=0.066$ ) (Figure $3 A, B, C$ ).

\section{Association between S100A8 and S100A9 expression and disease-free survival}

Five studies that included 735 cancer patients reported the relationship of S100A8 and S100A9 expression with recurrence-free survival (RFS), disease-free survival (DFS), and progression-free survival (PFS). Nicklas et al. (13) reported the HRs related to RFS and PFS in non-muscleinvasive bladder cancer. In this analysis, RFS and PFS were regarded as DFS, and the HRs reported by Nicklas et al. (13) were included.

The pooled HR was assessed using the random-effects model because of significant heterogeneity $\left(\mathrm{I}^{2}=65.9 \%\right.$, $\mathrm{P}=0.007)$. The pooled HR was 1.98 (95\% CI: $1.20-3.29$, $\mathrm{P}=0.008)$ ( $\mathrm{P}$ value is not shown in the figure), which indicated a significant association between high expression of S100A8 and S100A9 and poor DFS in patients with cancer (Figure 4). Subgroup analysis demonstrated that protein type $\left(\mathrm{S} 100 \mathrm{~A} 9, \mathrm{I}^{2}=66.5 \%, \mathrm{P}=0.050\right)$ and cancer type (non-urinary system cancer, $\mathrm{I}^{2}=76.8 \%, \mathrm{P}=0.013$ ) may be the causes of heterogeneity (Figure $5 A, B, C$ ).

\section{Sensitivity analysis}

Sensitivity analysis suggested that the results from Koh et al. (12) had a significant impact on the outcomes. However, the pooled HR did not prominently change after excluding each study one by one, which indicated that our results were consistent and robust (DSS, HR 1.48, 95\% CI: 1.07-2.04; DFS, HR 1.77, 95\% CI: 1.35-2.33; Figure 6A,B).

\section{Publication bias}

The funnel plot showed asymmetry (Figure $7 A, B$ ). This presents the possibility of publication bias, although 


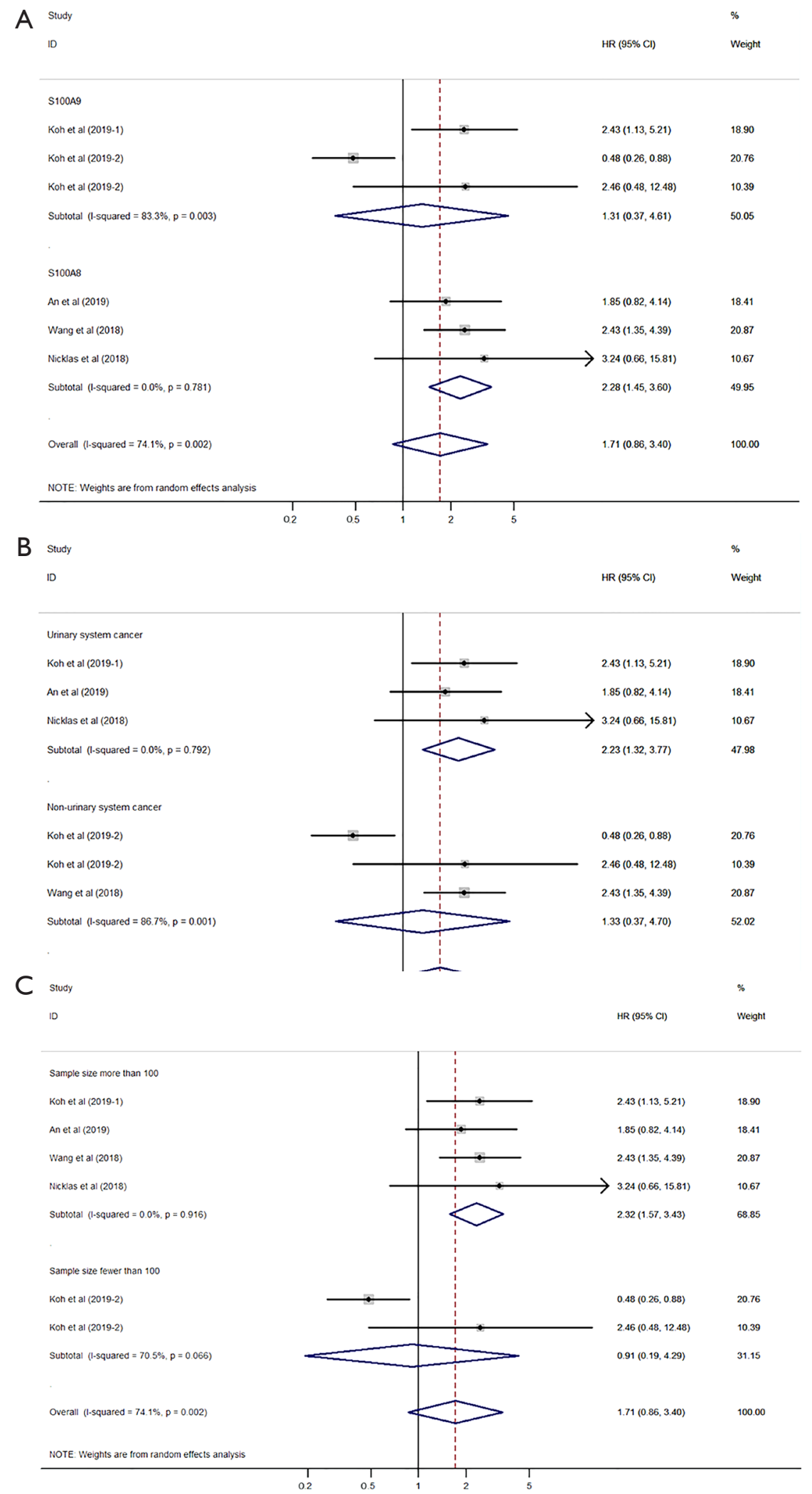

Figure 3 Forest plot for disease-specific survival subgrouped by protein type (A), cancer type (B), and sample size (C). 


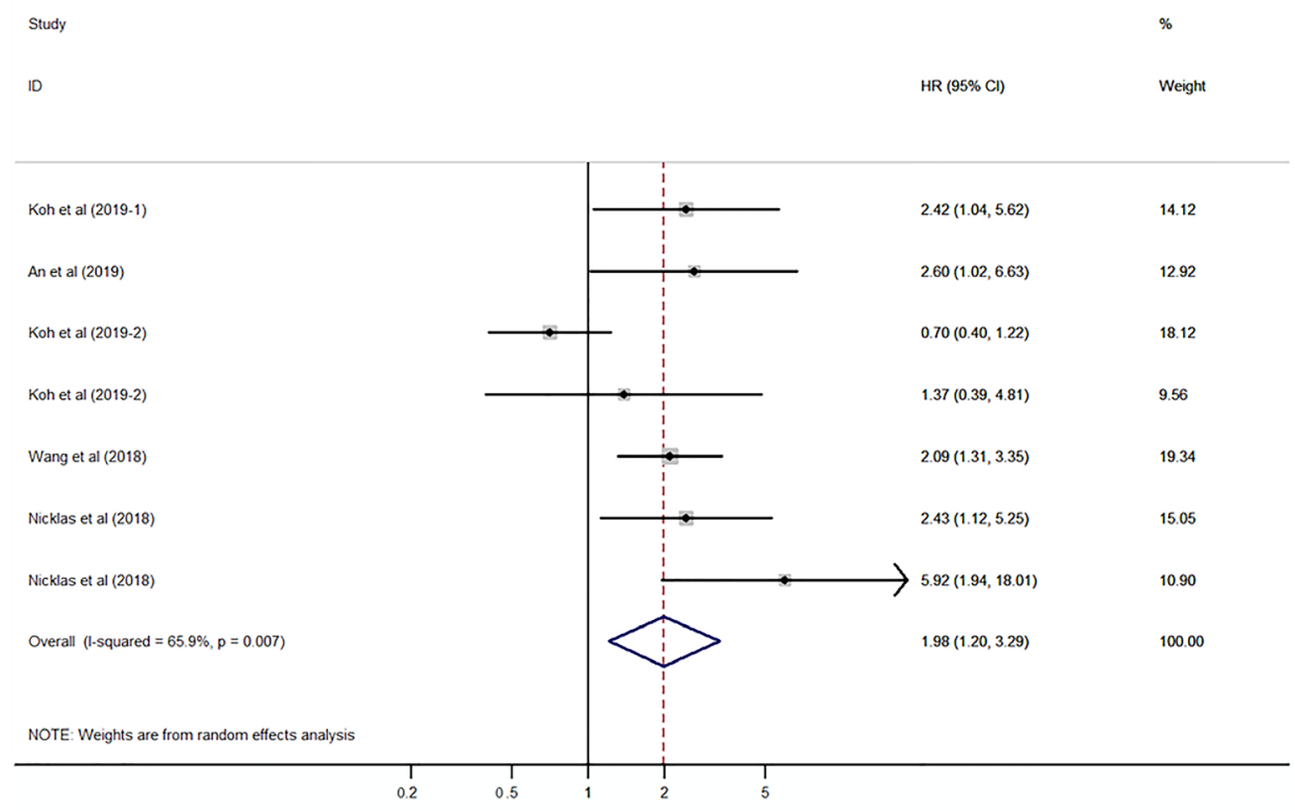

Figure 4 Forest plot presenting the association of S100A8 and S100A9 expression with disease-free survival in cancer patients.

Egger's regression test was not significant (DSS, $\mathrm{P}=0.470$; DFS, $\mathrm{P}=0.347)$. Thus, we conducted the trim and fill method. The test also did not change our initial results (Figure 7C,D).

\section{Discussion}

Calprotectin (S100A8/S100A9), a heterodimer of the two calcium-binding proteins S100A8 and S100A9, was primarily discovered as an immunogenic protein expressed and secreted by neutrophils (14). It has become evident as a valuable pro-inflammatory mediator in inflammation (14). In fact, S100A8 and S100A9 are considered biomarkers for inflammatory diseases, including multiple sclerosis, inflammatory bowel disease, rheumatoid arthritis, psoriasis, and cystic fibrosis (9). The relationship between inflammation and tumorigenesis has been identified (9). Overexpression of S100A8 and S100A9 has been found in inflammatory lesions associated with tumorigenesis (9). More recently, upregulation of S100A8 and S100A9 expression was reported in various human cancers, such as lung, gastric colon, prostate, breast, and skin cancers, with high expression in cancer cells $(6,9)$. Furthermore, S100A8 and S100A9 have been clearly recognized as new prognostic candidates playing important roles in regulating tumor cell growth, invasion, and metastasis (9). Therefore, we performed this meta-analysis to systematically understand the prognostic role of S100A8 and S100A9 expression in patients with cancer.

For this meta-analysis, we identified 5 eligible articles that included cancer patients. Koh et al. (15) revealed that S100A9 could be an important prognostic factor for poor DSS and DFS, and An et al. (16) showed that high expression of S100A8 was associated with poor DFS in patients with clear cell renal cell carcinoma. Koh et al. (12) also reported that S100A8 and S100A9 expression was associated with survivability in patients with squamous cell carcinoma and adenocarcinoma of the lung. Wang et al. (17) demonstrated that patients with increased S100A8 expression levels in breast cancer had significantly shorter DFS and OS. Nicklas et al. (13) reported that S100A8 expression was a promising marker for the identification of non-muscle-invasive bladder cancer patients who are at high risk of progression and recurrence.

In this meta-analysis, we showed that high expression of S100A8 and S100A9 was associated with poor DFS in patients with cancer. The pooled HR for S100A8 and S100A9 expression was 1.98 (95\% CI: $1.20-3.29, \mathrm{P}=0.008$, $\mathrm{P}$ value is not shown in Figure 4) for DFS. However, we did not find that the expression of S100A8 and S100A9 was significantly correlated with DSS (HR 1.71, 95\% CI: $0.86-3.40, \mathrm{P}=0.128, \mathrm{P}$ value is not shown in Figure 2). Furthermore, sensitivity analysis demonstrated that our results are consistent (DFS, HR 1.77, 95\% CI: 1.35-2.33). 


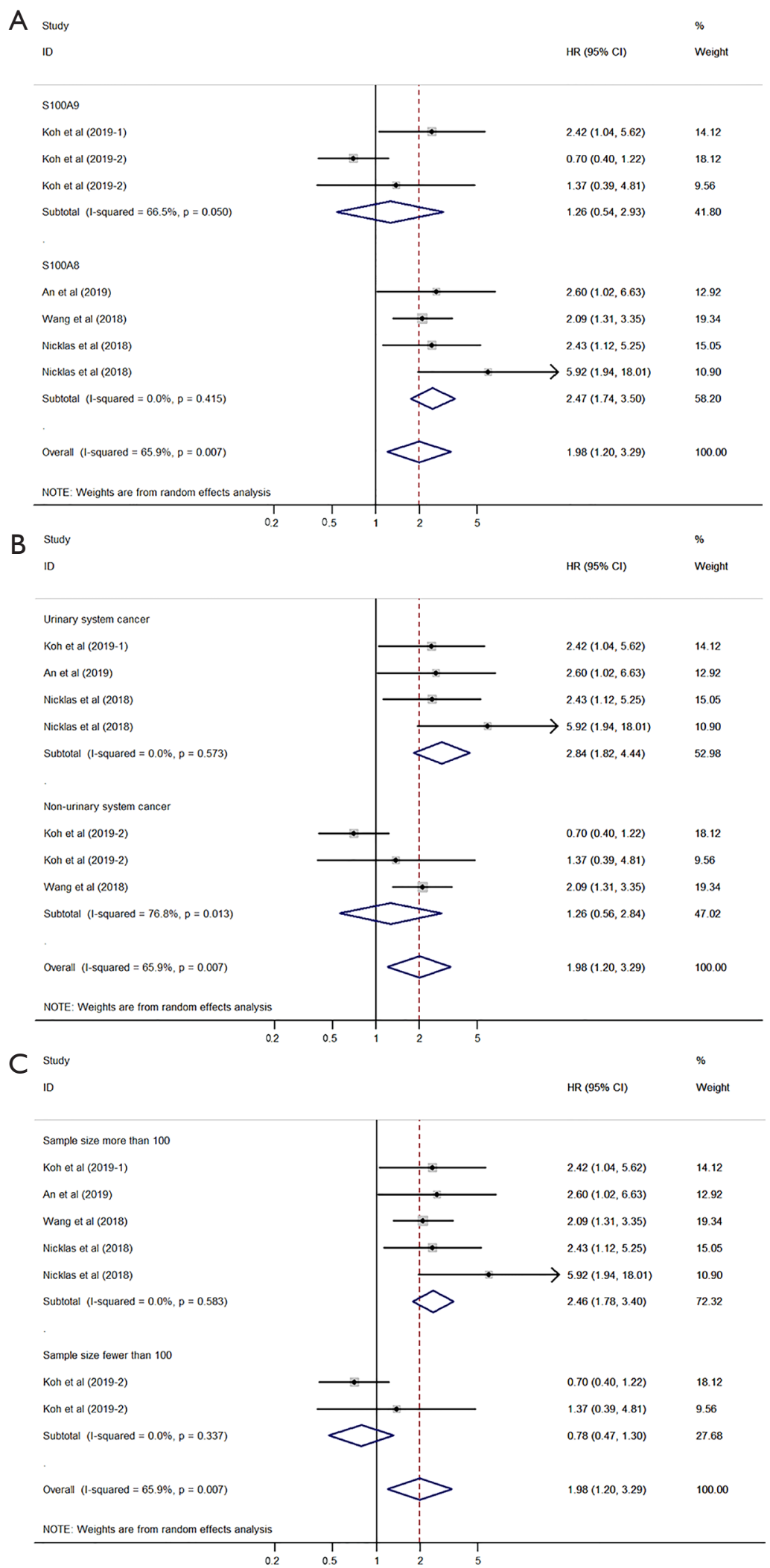

Figure 5 Forest plot for disease-free survival subgrouped by protein type (A), cancer type (B), and sample size (C). 

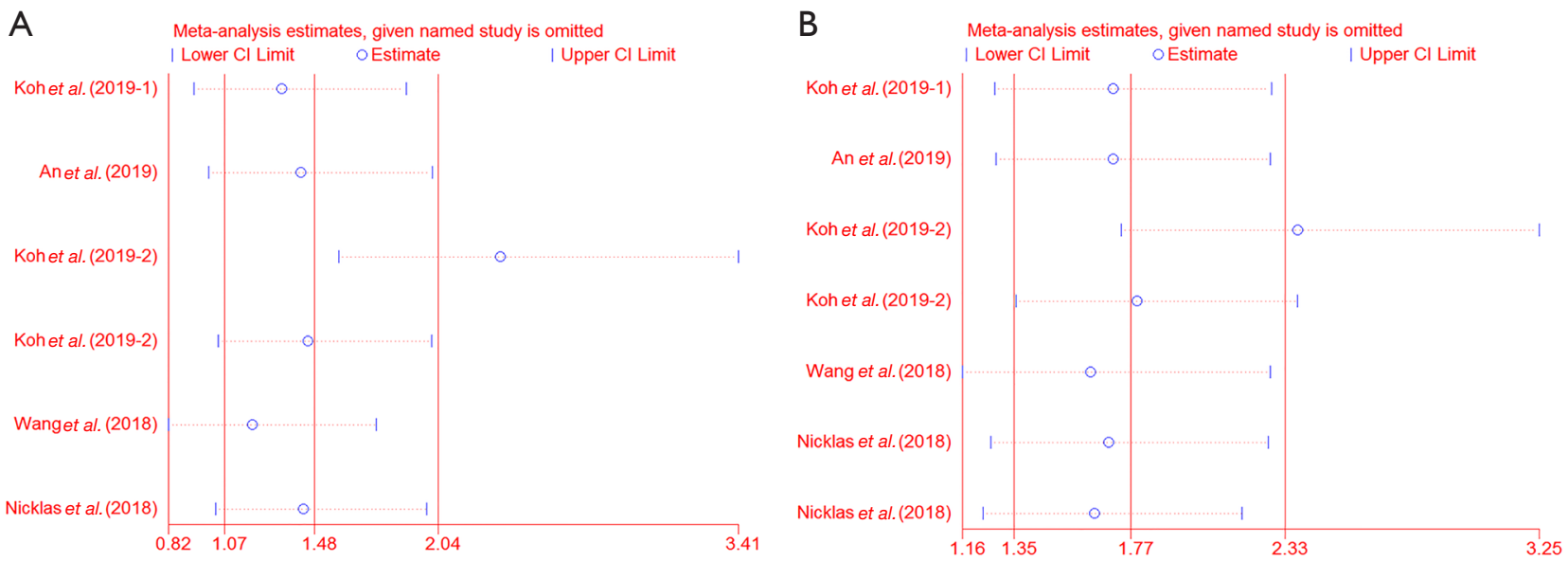

Figure 6 Sensitivity analysis for disease-specific survival (A) and disease-free survival (B).
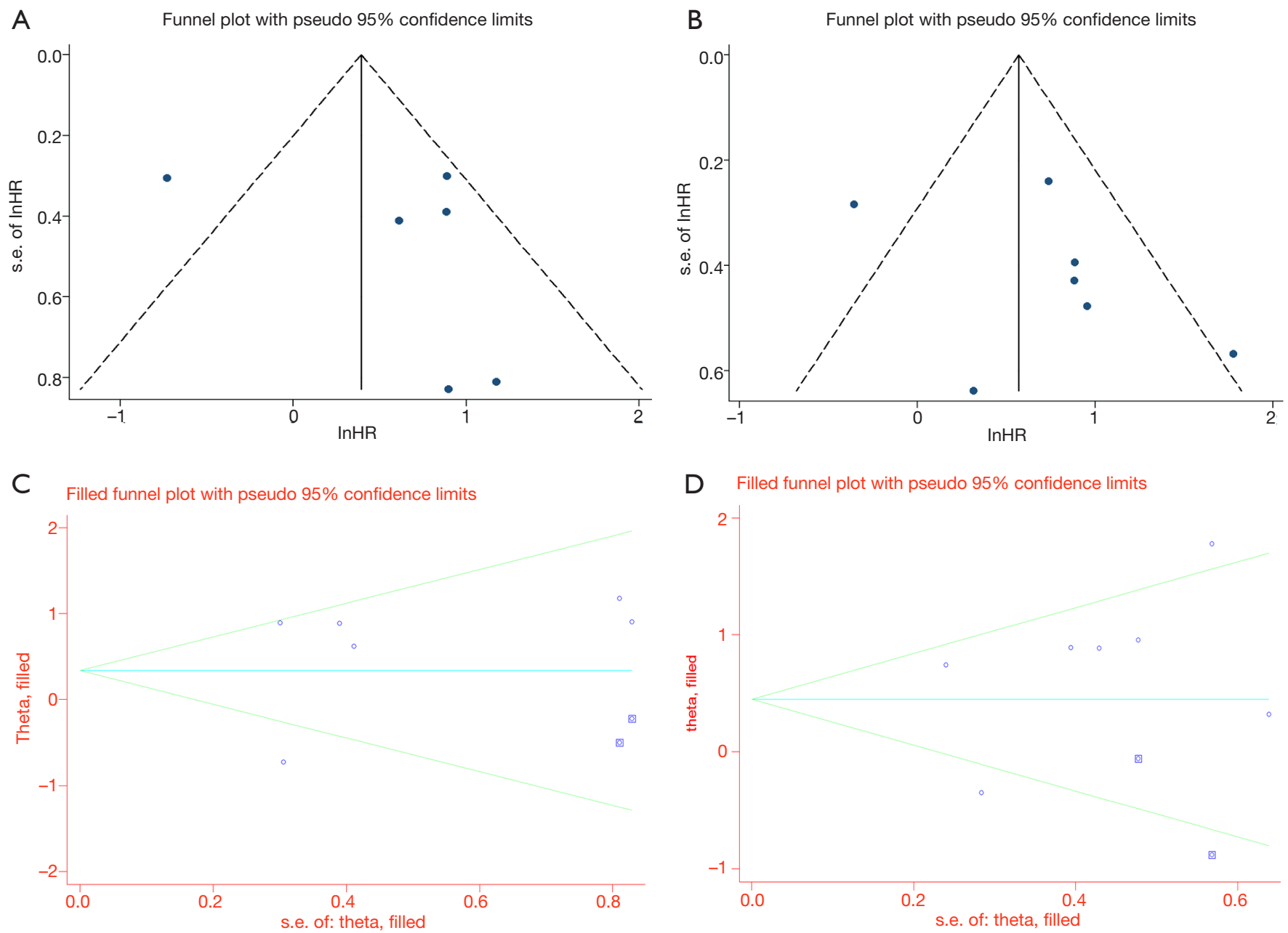

Figure 7 Funnel plot and trim and fill method for disease-specific survival (A,C) and for disease-free survival (B,D). 
Thus, S100A8 and S100A9 expression may be a potential prognostic biomarker of DFS in cancer patients. To the best of our knowledge, the current study is the first systematic review and meta-analysis to focus on survival in cancer patients based on S100A8 and S100A9 expression.

This meta-analysis has several limitations. First, some results had heterogeneity, although the random-effects model was used and subgroup analysis was performed, which may have caused bias in the results. Second, the cutoff value for S100A8 and S100A9 expression varied among the included articles, which may have caused heterogeneity in the results. Finally, the sample size of the included articles was small, which could have resulted in a bias in the outcome.

In conclusion, the current systematic review and metaanalysis showed that high expression of S100A8 and S100A9 may be a poor prognostic marker of DFS in cancer patients.

\section{Acknowledgments}

Funding: This work was supported by the Soonchunhyang University Research Fund; biomedical research institute fund (GNUHBRIF-2021-0005) from the Gyeongsang National University Hospital.

\section{Footnote}

Reporting Checklist: The authors have completed the PRISMA reporting checklist. Available at https://dx.doi. org/10.21037/tcr-21-519

Conflicts of Interest: All authors have completed the ICMJE uniform disclosure form (available at https://dx.doi. org/10.21037/tcr-21-519). The authors have no conflicts of interest to declare.

Ethical Statement: The authors are accountable for all aspects of the work in ensuring that questions related to the accuracy or integrity of any part of the work are appropriately investigated and resolved.

Open Access Statement: This is an Open Access article distributed in accordance with the Creative Commons Attribution-NonCommercial-NoDerivs 4.0 International License (CC BY-NC-ND 4.0), which permits the noncommercial replication and distribution of the article with the strict proviso that no changes or edits are made and the original work is properly cited (including links to both the formal publication through the relevant DOI and the license). See: https://creativecommons.org/licenses/by-nc-nd/4.0/.

\section{References}

1. Han L, Wang B, Wang R, et al. Prognostic and Clinicopathological Significance of Long Non-coding RNA PANDAR Expression in Cancer Patients: A MetaAnalysis. Front Oncol 2019;9:1337.

2. Hua X, Chen J, Wu Y, et al. Prognostic role of the advanced lung cancer inflammation index in cancer patients: a meta-analysis. World J Surg Oncol 2019;17:177.

3. Fan $\mathrm{H}$, Wang W, Yan J, et al. Prognostic significance of CXCR7 in cancer patients: a meta-analysis. Cancer Cell Int 2018;18:212.

4. Arai K, Takano S, Teratani T, et al. S100A8 and S100A9 overexpression is associated with poor pathological parameters in invasive ductal carcinoma of the breast. Curr Cancer Drug Targets 2008;8:243-52.

5. Fan B, Zhang LH, Jia YN, et al. Presence of S100A9positive inflammatory cells in cancer tissues correlates with an early stage cancer and a better prognosis in patients with gastric cancer. BMC Cancer 2012;12:316.

6. Salama I, Malone PS, Mihaimeed F, et al. A review of the S100 proteins in cancer. Eur J Surg Oncol 2008;34:357-64.

7. Yasar O, Akcay T, Obek C, et al. Significance of S100A8, S100A9 and calprotectin levels in bladder cancer. Scand J Clin Lab Invest 2017;77:437-41.

8. Choi DK, Li ZJ, Chang IK, et al. Clinicopathological roles of S100A8 and S100A9 in cutaneous squamous cell carcinoma in vivo and in vitro. Arch Dermatol Res 2014;306:489-96.

9. Srikrishna G. S100A8 and S100A9: new insights into their roles in malignancy. J Innate Immun 2012;4:31-40.

10. Hiratsuka S, Watanabe A, Aburatani H, et al. Tumourmediated upregulation of chemoattractants and recruitment of myeloid cells predetermines lung metastasis. Nat Cell Biol 2006;8:1369-75.

11. Lim SY, Yuzhalin AE, Gordon-Weeks AN, et al. Tumor-infiltrating monocytes/macrophages promote tumor invasion and migration by upregulating S100A8 and S100A9 expression in cancer cells. Oncogene 2016;35:5735-45.

12. Koh HM, An HJ, Ko GH, et al. Prognostic Role of S100A8 and S100A9 Protein Expressions in Nonsmall Cell Carcinoma of the Lung. J Pathol Transl Med 
2019;53:13-22.

13. Nicklas AP, Kramer MW, Serth J, et al. Calgranulin A (S100A8) Immunostaining: A Future Candidate for Risk Assessment in Patients with Non-Muscle-Invasive Bladder Cancer (NMIBC). Adv Ther 2018;35:2054-68.

14. Gebhardt C, Németh J, Angel P, et al. S100A8 and S100A9 in inflammation and cancer. Biochem Pharmacol 2006;72:1622-31.

15. Koh HM, An HJ, Ko GH, et al. Prognostic role of S100A9 expression in patients with clear cell renal cell carcinoma. Medicine (Baltimore) 2019;98:e17188.

16. An HJ, Koh HM, Song DH. S100A8 Expression May Have a Prognostic Value in CCRCC Reflecting TNM Staging and Fuhrman Nuclear Grade. Anticancer Res 2019;39:4681-5.

17. Wang D, Liu G, Wu B, et al. Clinical Significance of Elevated S100A8 Expression in Breast Cancer Patients. Front Oncol 2018;8:496.
Cite this article as: Koh HM, Lee HJ, Kim DC. High expression of S100A8 and S100A9 is associated with poor disease-free survival in patients with cancer: a systematic review and meta-analysis. Transl Cancer Res 2021;10(7):3225-3235. doi: $10.21037 /$ tcr-21-519 